\title{
A Method for Information Systems Testing Automation
}

\author{
Pedro Santos Neto ${ }^{1,2}$, Rodolfo Resende ${ }^{1}$, and Clarindo Pádua ${ }^{1}$ \\ 1 Computer Science Department, \\ Universidade Federal de Minas Gerais (UFMG) \\ \{pasn, rodolfo, clarindo\}@dcc.ufmg.br \\ 2 Computer Sciense and Statistic Departament, \\ Universidade Federal do Piauí (UFPI) \\ pasn@ufpi.br
}

\begin{abstract}
This paper presents MODEST, a MethOD to hElp System Testing. MODEST can reduce the overall effort required during software construction, using an extended design specification produced in a UPlike software process. This specification is used to automate test generation and execution, decreasing the effort required during test activities. The method deals with Information Systems that follow an architecture composed of a user interface layer, a business rule layer and a storage mechanism abstracted by a persistence layer.
\end{abstract}

\section{Introduction}

Information Systems (IS) have a very important role in our lives. They are usually constructed using many components that operate together in order to retrieve, process, store, and distribute information. This information is generally used to help the analysis, control and decision making in an organization. Because of this, Information Systems became a pervasive technology, i.e., they are more noticeable by their absence than their presence.

Considering the relevance of Information Systems, a flaw during their operation can cause big damages. According to a report produced by NIST 11], the USA annual costs, due to an inadequate infrastructure for software testing, are estimated to range from $\$ 22.2$ to $\$ 59.5$ billion, only in 2002 . Over half of these costs involve error avoidance and mitigation activities. The remaining costs involve additional testing resources that are consumed due to inadequate testing tools and methods. Many of these flaws are related to IS development.

These problems motivate our investigation on how to improve the efficiency of test activities, in particular the ones related to the following IS features:

- The ability to manage large amounts of persistent data.

- The management of a large number of users accessing information.

- The utilization of graphical user interfaces.

- The integration with other enterprise applications. 


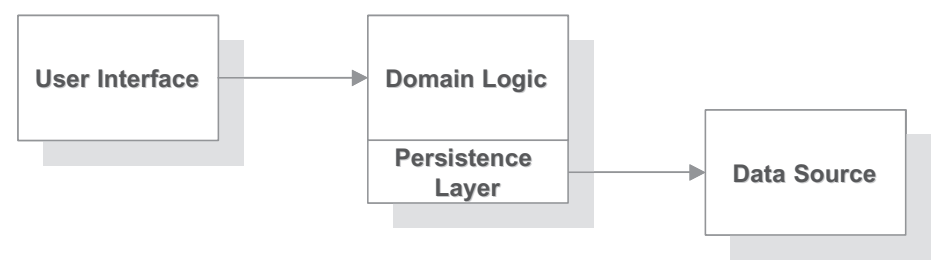

Fig. 1. The architecture supported by MODEST

In this work we present the MethODod to hElp System Testing (MODEST). The main purpose of this method is the automatic generation and execution of system testing. This method takes advantage of the artifacts created during the development of an application. Most of the concepts in the method are process and modeling language independent, nevertheless, for the sake of readability and pragmatism, we describe MODEST in the context of the Unified Process - UP [2] guidelines. We use the UML [3] as prescribed by UP.

Our current work deals with softwares composed by a presentation layer, a business rule layer, and a storage mechanism abstracted by a persistence layer, as informally shown in Figure 1. This organizational structure is captured within the architecture of many Information Systems.

The remainder of the paper is organized as follows. Section 2 presents the method, detailing each activity. Section 3 briefly describes a MODEST compliant tool. Section 4 discusses an experimental study evaluating the method. Section 5 describes some related works. Section 6 presents our conclusions and future works.

\section{MODEST Overview}

The main objective of MODEST is to reduce the effort required in the system testing activities. Most aspects of the method are process independent, despite the use of several design artifacts produced during the system construction. Processes based on the UP process frequently create the artifacts used by MODEST. They are created using UML and it is simple to introduce the formalism required by the method.

Since we are interested in system testing, we use internal and external specification. We consider external specification the use case view of the system, showing the interactions among the system and the outside actors, without revealing the system internal structures. The internal specification corresponds to the logical view of the system and shows how to implement the system behavior in an effectively computable way [3].

Figure 2 shows the method activities. Activities A1 to A6 are start up activities. These activities detail how the software under test (SUT) should work. The method uses the information gathered in these activities to generate and execute tests, verifying if the software follows the specification. We assume that 


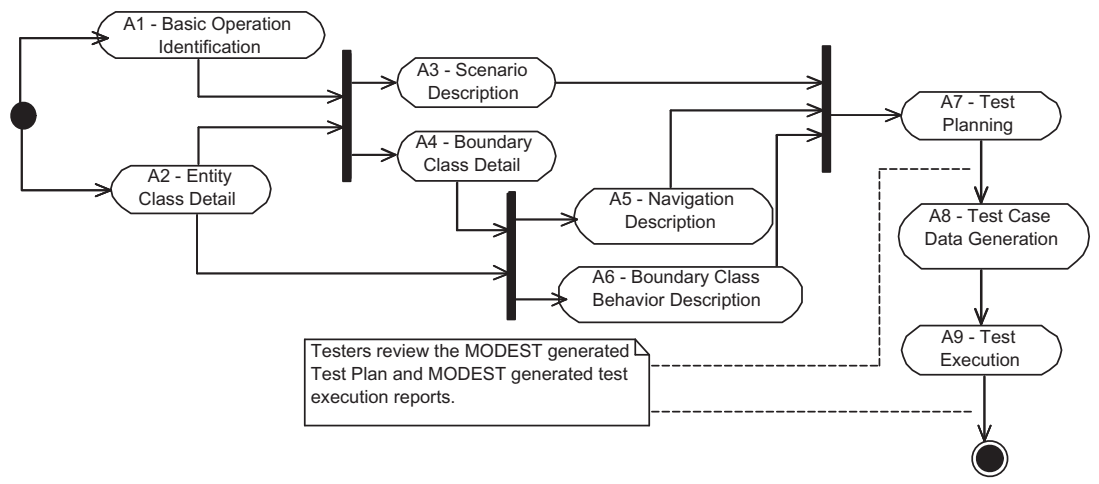

Fig. 2. Activity diagram showing MODEST activities

the specification serving as input to MODEST is correct, however the method pescribes several consistency checks as will be seen later. The correction of the specification can be assured by reviews, inspections or other techniques.

Activities A7 to A9 generate test procedures and test cases, execute the tests and create reports to be analyzed by the testers.

In the following subsections we briefly discuss each activity. We use a user authentication protocol as a running example. This simple example is composed by two use cases: Login and User Management. There are three windows in this example: Main Window, used to authenticate users, UsersWindow, used to manage users (create, read, update, delete), and SearchWindow, used to search an element, in this case Users, in a collection.

In some sentences of this work, written as "MODEST uses the information", we are referring to MODEST as a tool and not a method description. We use this style since a method induces a corresponding tool, and in some places it is easier to explain how a MODEST compliant tool works, instead of describing the method prescriptions. We discuss a MODEST compliant tool in Section3.

\subsection{Basic Operation Identification}

MODEST uses the persistence mechanism to generate and verify the test results. These operations are related to one of the CRUD (Create, Read, Update, Delete) operations usually provided by a persistence layer.

During test generation, it is necessary to know the CRUD operations to populate the storage mechanism with the data required in the test. During the test execution it is necessary to verify the result of each persistent operation used, thus, if the create operation is used, it is necessary to verify if the appropriate data was correctly stored.

There are many ways to identify the persistence operations in a system. In our work, we decided to adopt the use of diagrams describing the CRUD operations of the persistence mechanism. These diagrams follow some naming and formatting conventions, facilitating the retrieval of the required information. 
This option improves the quality of the project documentation, detailing how the persistence mechanism works.

\subsection{Entity Class Detail}

The entity classes usually model persistent information. These classes are usually independent of applications and they are strong candidates for reuse. MODEST uses these classes and their associations for storage mechanism population.

MODEST prescribes the specification of the entity classes and their associations, together with properties related to the entity class attributes. These extra properties must detail if an attribute is a key field, its type, valid values, minimal and maximal value, and if the attribute initialization is mandatory. As mentioned in Subsection 2.4, it is possible to create a link between an entity class attribute and a user interface field, in order to allow the test case generation.

There are some non mandatory entity classes properties that can be used for the generation of non-functional tests. If the query frequency, update frequency, maximum cardinality and maximum time for queries, are given MODEST prescribes how to populate the storage mechanism, and how to generate tests checking the time spent during CRUD operations.

\subsection{Scenario Description}

Scenarios illustrates a sequence of actions related to a behavior. In the UML, they are conventionally described using interaction diagrams. A scenario provides details about how to implement behavior in an effectively computable way. MODEST uses these scenarios, in order to discover what persistent operations are used and to help the determination of scenario expected result.

MODEST prescribes some modeling conventions for scenario description. First, each activated user interface command must be represented in the diagram by an operation with the same name. Second, these operations must receive, as parameters, the data required by its functioning. Third, there must be a mechanism for the identification of persistence operations used in a scenario. Fourth, all the exceptions thrown in the scenario must be detailed.

Figure 3 presents a scenario that follows MODEST prescriptions. Login scenario illustrates one of the Login use case executions. The first operation in the scenario corresponds to a user interface command (message number 1 in the diagram). The data required to execute this operation are the login and password. It is simple to identify the persistence operation invoked: there is a call to read method (message 7 ). Besides, all the exceptions related to this scenario are detailed, including error messages and the thrown conditions (messages 4, 6, 8 and 9).

MODEST prescribes how to create test procedures based commands activated in the scenario, the required data, and the executed persistence operations.

\subsection{Boundary Class Detail}

MODEST relies on boundary stereotyped classes representing user interfaces. These classes are central points for data input and output. 


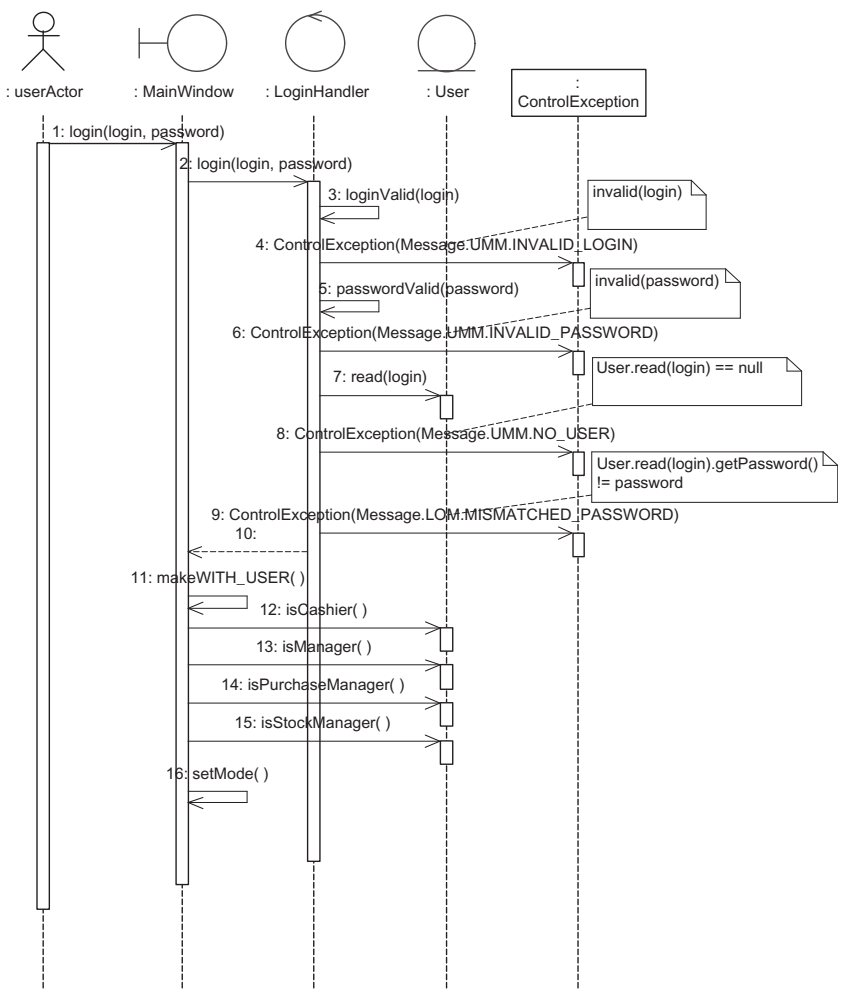

Fig. 3. Login scenario

MODEST prescribes the description of fields and operations visible by the end users. For instance, we modeled user interface fields in our example, using the stereotype $<<$ field $>>$ on attributes, and using the stereotype $<<$ command $>>$ on operations. MODEST recognizes this information and extracts the related data for use.

MODEST uses the specification of several relevant characteristics associated to fields and commands in order to generate and execute test cases. A field attribute must identify the style used in the target language (TextField, PasswordField, CheckBox, etc.), together with the source and the destination target of the field data. For instance, a source of a field can be an entity attribute, a software/hardware interface, a pre-defined value, e.g. system date, or a calculated value, e.g. the sum of two entity attributes. Fields related to an entity attribute must specify this association. This allows the automatic data generation, using the entity attributes properties.

A user interface command must also identify the style used in the target language (like Button or a MenuItem), the preconditions required for command activation, and postconditions specifying the system state after finishing the 
command execution. MODEST does not prescribe a specific language to create these conditions. Any language with suitable expressive power can be used.

In our example, we used a simple language based on the persistence layer operations. Currently, we do not use OCL [4], in order to simplify our investigations. We are planning to adopt OCL in future versions our work. Below, we present a simplified version of the grammar for our language. This grammar is used to specify all the conditions presented in our examples.

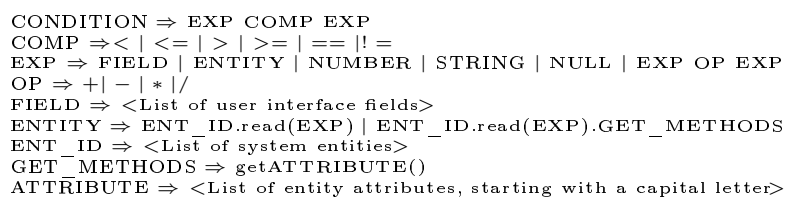

\subsection{Navigation Description}

MODEST prescribes the use of information related to user interface control transfer. This information can be represented in UML using a collaboration diagram involving the boundary class instances or by a class diagram with control transfer modeled by associations stereotyped as $<<l i n k>>$.

Figure 4 shows control transfers of part of our example. Associations between boundary classes should be stereotyped with $\langle<$ link $>>$ whenever the association represent a possible navigation. Constraints on these associations are shown inside curly braces together with the corresponding commands. The control transfer from Users Window to Search Window objects occurs only when the search button is clicked and the login field is void (login $==" \prime \prime)$. MODEST specifies that all the constraints presented in this description must be exercised, i.e., used to create a test case.

\subsection{Boundary Class Behavior Description}

The expected behavior of the IS is directly related to the interaction of users with their user interfaces. We assume that in a typical design the exceptions are always associated with user interface error messages. We also assume that exceptions are modeled using the presentation of error messages conditions.

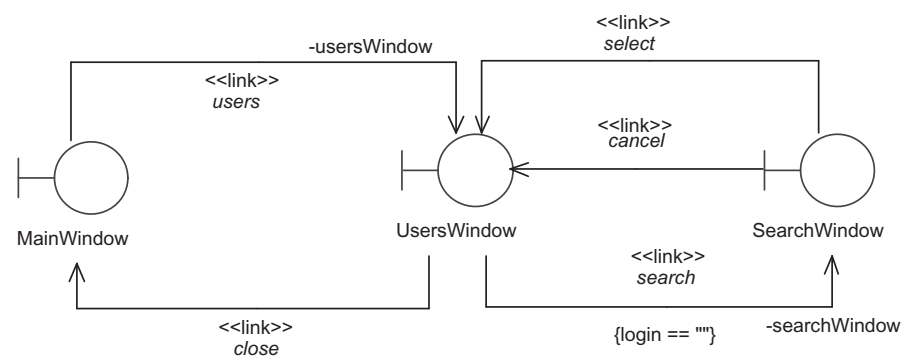

Fig. 4. A diagram showing the possible control transfers among windows 


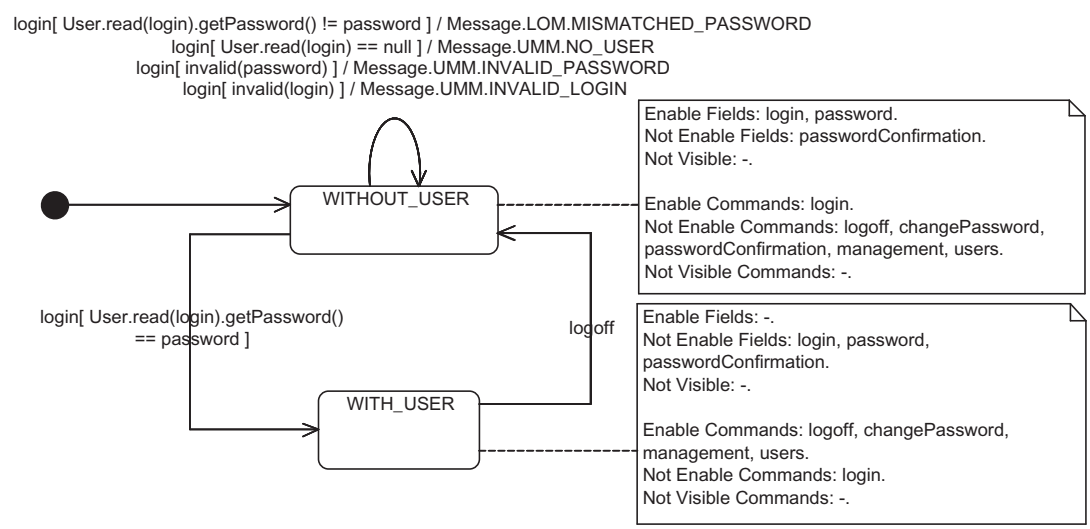

Fig. 5. Main Window statechart excerpt

MODEST heavily uses the descriptions of the boundary classes behavior. Using this information it is possible to determine what needs to be tested and how to test it. However, it is necessary to provide these descriptions in a suitable format.

Statechart diagrams can be used to represent the boundary class behavior. Each boundary class needs to have a diagram showing the normal behavior and exceptions. The exceptions are modeled as guard conditions. Figure 5 shows an example of these descriptions for the MainWindow of our example. The user uses this window to login into the system. In order to have this user interface testable by using the method, we need to describe all the possible exceptions and user interface presentations.

The initial state of MainWindow is WITHOUT USER. This state describes one user interface presentation, indicating the enabled, disabled and invisible fields and commands. The user interface presentation needs to be tested after each command execution. All the exceptions related to WITHOUT_USER are modeled in the statechart of the Figure 5. For instance, when the user clicks on the login button and the informed password does not match with the stored password, a message describing this is showed (Messages.LOM.MISMATCHED-

PASSWORD). This is represented by the first self-transition for the WITHOUT_USER in Figure 5.

MODEST prescribes many consistency checks of the specification before test generation and execution. For instance, the login command must be enabled in the WITHOUT USER state, described in Figure 5, since there are many transitions related to this command. Besides, since there are some exceptional conditions also related to this command, these conditions must be described in every scenario that uses it, beyond the statecharts. There are many other consistency rules prescribed by MODEST, but we do not describe them here in order to save space. 


\subsection{Test Planning}

In this activity, MODEST defines the test cases, indicating the associated test procedures and the conditions to be exercised. The test case data generation is done in the next activity.

MODEST will deal with every state and transition in the statecharts, and all the exceptions and scenarios.

Algorithm 1 depicts, in a simplified way, how MODEST defines the test case sequences. The general idea is always to search for any unused specification, in order to exercise it.

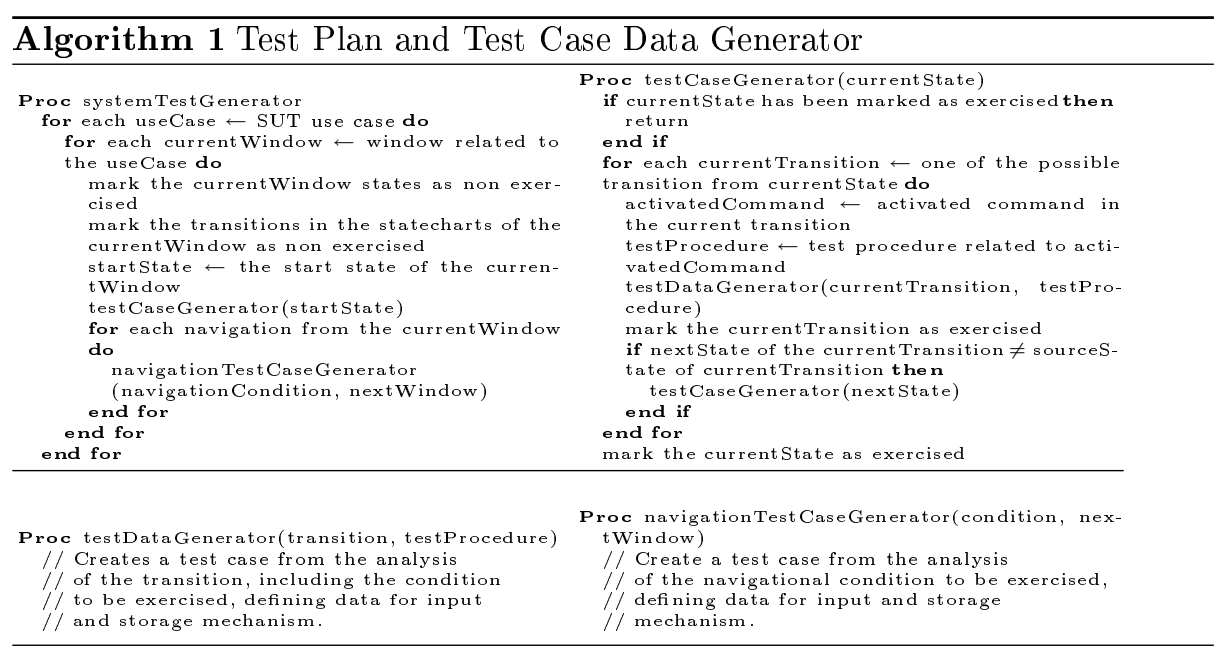

\subsection{Test Case Data Generation}

After the determination of the test case sequences, it is necessary to specify the test input and output data. This is the main goal of this activity.

The test case inputs are determined analyzing the transition to be exercised, together with the properties of the involved fields. For instance, after the generation of test case sequences for our example, it is generated a test case related to the test procedure Login, based on the Login scenario, to exercise the first self-transition of Figure 5. In this transition there is a condition "User.read(login).getPassword() != password". This condition was created using our simple language, defined previously. MODEST prescribes the analysis of this condition, in order to generate input and persistent data.

In this example, it is necessary to generate a User with a valid login, in the storage mechanism, but with a password different from the stored password. MODEST prescribes the generation of valid values, using the properties specified in the user interface fields. As mentioned before, MODEST prescribe the specification of properties related to a user interface $<<$ field $>>$ indicating its source and target. The login field of our example is related to the login attribute 
of User entity. This field is a key field, with minimal size two, maximal size eight, composed only by letters. So, it is simple to generate a valid login and password. MODEST also prescribes the determination of the persistent data required to run the test cases. In this example, it is required the existence of a specific User. The data fields required to this test case execution must the determined in this activity. An example is the creation of a User with a login "aaaa", password "aaaa". The test case data could be login "aaaa" and password "bbbb". This would assure the use of the desired condition ("User.read(login).getPassword() $!=$ password").

At the end of this activity, several definitions are available: the input and persistent data required to run the test case, the conditions to be evaluated before and after each command activation, and the expected reached state after each command activation. Instead of specifying the determination of the output data, MODEST specifies how to express the conditions that must be satisfied after a command execution. This option has a low cost with reasonable benefits, compared to output data specification. These conditions are defined in the boundary class detail activity, and they are related to command pre and postconditions, together with the persistence operations used in the scenario related to the test procedure. These characteristics facilitate the determination of a test verdict for the test case, as we can see in the next activity.

\section{$2.9 \quad$ Test Execution}

MODEST prescribes the automatic test case execution. Many technologies can be used for this, such as computational reflection [5] and aspects [6].

Using reflection, for instance, it is possible to discover all the class attributes and operations during runtime, without requiring code changes. This information, combined with the boundary class description, can be used by a tool that obeys MODEST to put test case data in the appropriated fields and to execute the commands required in the test procedures.

In order to allow automatic test case execution, MODEST prescribes some conventions to be used in attributes and operations names. This is required in order to facilitate the identification of user interface fields and commands.

Executing a test case requires the storage mechanism population for the test, boundary classes loading, instantiation of user interfaces, setting of the appropriate data into the fields, and the execution of the commands in the corresponding test procedures, always checking the pre and postconditions, the reached state and the expected result related to the persistence operations.

MODEST prescribes the determination of a test verdict at the end of the test case execution. This verdict follows the UML Testing Profile specification [7]. There are four valid values: fail, inconclusive, pass, and error. A pass verdict indicates that the test case is successful and that the SUT has behaved as expected. A fail verdict on the other hand shows that the SUT is not behaving according to the specification. An inconclusive verdict means that the test execution cannot determine whether the SUT performs well or not. An error verdict tells that the test system itself and not the SUT failed. 


\section{A MODEST Compliant Tool}

We developed a prototype to function as a MODEST compliant tool, named MODESToo. We show the MODESToo components in Figure 6. MODESToo reads an XMI [8] specification to extract the data related to software behavior. $\mathrm{XMI}$ is a standard format for metadata interchange for UML Tools. This format allows the interoperation of UML tools, provided they are able to generate an XMI file containing the software specification.

The Extractor is a MODESToo component responsible for data extraction. During this task, a consistency check is done, and the test procedures are generated based on the scenario descriptions.

The Test Planner generates the test case sequences to test the system. This component creates the test case sequences, detailing the test procedure to be used, the reached states during the test procedure execution, the conditions to be exercised, and the pre and postconditions to be verified.

The Test Case Data Generator is responsible for input data generation, based on the condition to be tested. It is also responsible to determine the persistent data required during the test case execution. The current Test Case Data Generator is based on our language, defined in the Subsection 2.3.

The Populator is the component responsible for storage mechanism population, based on the requirements of the test case. This can be a complex task, since an entity can have many associations and many mandatory fields not specified by the Test Case Data Generator. The Test Case Data Generator specifies the fundamental data for the test. For instance, if the Test Case Data Generator specifies the creation of a User with password "123456", the Populator must generate a User with a login, a password, a name, and belonging to some groups, since these properties are mandatory for a User creation. The password is the fundamental data for this example, and the other values are arbitrary.

The Executor is responsible for the system load, data input, and result analysis. It calls the Populator before each test case execution, to start a transaction and to create the persistent data required for test execution. This transaction is terminated with a roll back, at the end of the test, in order to remove all the

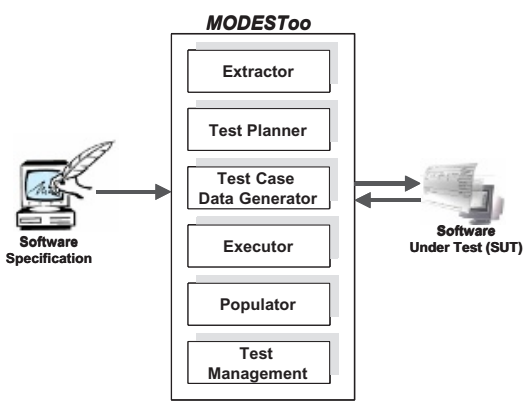

Fig. 6. A MODEST compliant tool 
operations effects. This is the only component dependent on technology: if the SUT is a Web based Information System, it is required a Web Executor, if the SUT is a Java-GUI based Information System, it is required a Java Executor. All the conditions related to the test case must be evaluated by this component. The Executor is responsible for the test case verdict generation. It also stores the output test case data for future visualizations.

The Test Management is a graphical user interface for MODESToo administration. This component is used to start the method and to visualize the generated tests together with their execution. Besides, there are some facilities for test cases creation, decreasing the effort required in this task.

\section{Experimental Study}

We developed a study with the motivation to assess and better understand the use of MODEST. The purpose of the study was to evaluate, from the perspective of developers, the use of a software process instantiated with and without MODEST. We refer to these processes here as MEP - MODEST Enhanced Process, and NEP - Non-Enhanced Process. MEP and NEP processes are based on the UP software process. We adopted the recommendations of Basili, Selby, and Hutchens [9] in this study.

We started the experiment with sixteen software engineering students, along the experiment ten students dropped out, and only six of them finished the activities. We believe that the free character of the voluntarism can explain this. Three volunteers used MEP and three others used NEP to develop the Login use case discussed before. Table 1 shows the effort required by the two groups of students. The design specification was created using Rational Rose [10] modeling tool. Volunteers using MEP dedicated lower effort than NEP developers, since MODESToo automatically generates a partial test plan, including the test procedures and the test cases, based on the design specifications. Developers using NEP had an extra work to generate a test plan and to implement the test cases using Abbot [11] tool. In the experiment we considered design and test efforts.

Both groups had a four-hour explanation on the techniques involved in the experiment. The MEP group had an extra four-hour class detailing the MODEST conventions required in the design. The artifacts generated by the developers were reviewed by the paper authors.

Our experiment was designed with the goal of answering the following main questions:

Table 1. Effort spent in the experiments (in hours)

\begin{tabular}{|c|c|c||c|c|c|}
\hline \multicolumn{3}{|c|}{ MODEST } & \multicolumn{3}{c|}{ NORMAL } \\
\hline Dev1 & Dev2 & Dev3 & Dev4 & Dev5 & Dev6 \\
\hline 3,50 & 5,32 & 3,72 & 9,75 & 18,47 & 20,31 \\
\hline
\end{tabular}




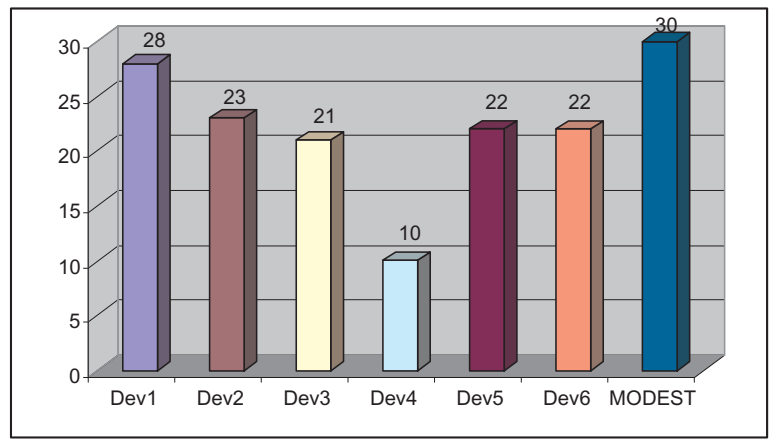

Fig. 7. Number of failures detected per developer during experimental study

- What is the extra time required to create a software design model extended with MODEST prescriptions?

- Is it possible to decrease the construction effort using MODEST?

- What is the quality of the MODEST generated tests?

The experiment analyzed the following two hypotheses: (i) MEP design specification creation effort is the same as in NEP, and (ii) MEP overall effort is the same as in NEP.

Hypothesis (i) was confirmed, and hypothesis (ii) was considered false, from the analysis of the collected data (95\% confidence interval). In other words, the extra-time required by MODEST extensions was compensated by the time gained in test activities, and the overall effort reduction was significant.

Additionally, we asked all the volunteers, MEP and NEP, to build a test plan and to implement this test plan, in order to evaluate the quality of MODEST generated tests, even though MEP volunteers were not supposed to deal directly with tests. From past software engineering classes student projects we retrieved the thirty most common failures. We injected a fault for each of these failures in the experiment SUT. Figure 7 shows the number of detected failures per developer, using the manually generated tests, and MODEST generated tests. We concluded, from this figure that there are failures detected by MODEST and not detected by the volunteers, therefore the failures detected by MODEST are not trivial. Volunteers detected an average of 21 faults, with a standard deviation of 5.93. Each injected fault was detected by at least one of the manually generated tests, showing that injected faults were not specially contrived.

\section{$5 \quad$ Related Work}

Offutt and Abdurazik [12] claim to be the first group to formalize a testing technique based on UML. They created a technique for test data generation based on statecharts. This technique was innovative but difficult to deal with systems containing many classes with many concurrent statecharts. 
Briand and Labiche [13] developed a method for functional system testing named TOTEM (Testing Object-orienTed systEms with the unified Modeling language). This method derives test requirements from artifacts created during analysis. It requires the creation of an activity diagram showing the use case dependencies to allow the test generation. They do not describe completely how to generate the test cases, how to start the tests if some data is required, and how to reduce, effectively, the huge amount of generated tests. They do not report a TOTEM compliant tool and method evaluation.

The AGEDIS [14] project created a methodology and tools for automated model driven test generation and execution for distributed systems. The project includes an integrated environment for modeling, test generation, test execution, and other test related activities. Their own work point out some problems. These problems are related to the modeling language conventions, the use of statechart as the main behavioral description of the SUT, and the language used as action language. We believe that another problem is the use of some uncommon artifacts for test generation, like the test generation directives.

In the Siemens Research Center was developed an integration testing technique for components using UML statecharts [15]. This method requires the definition of the dynamic functioning of the components using statechart diagrams adopting CSP (Communicating Sequential Processes) for describing the communication between two components. They do not report how their technique blends with a software process.

IRISA developed a method and a tool for automatic generation of test cases from UML design specification [16]. The UML model describing the system must have a class diagram showing the class associations, a object diagram showing the start configuration and a statechart for each class. The test objectives must be specified in sequence diagrams with some restrictions that can make their creation hard. Their work lacks some directives, for example, there is no comprehensive description about how the start configuration is set up during the test executions.

\section{Conclusion}

This paper presents MODEST, a MethODology to hElp System Testing. MODEST can reduce the overall effort required during software construction, using an extended design specification usually produced by software processes that follow the UP guidelines. This specification is used to automate test generation and execution, decreasing the effort required during test activities. The method deals with Information Systems composed by a presentation layer, a business rule layer, and a storage mechanism abstracted by a persistence layer, as informally shown in Figure 1.

Adapting MODEST for use in a UP-like software process was simple. We extended some activities and the registered impact was not significant, as mentioned in hypothesis (i) of Section 4. Besides, the overall effort reduction was significant, since MODEST automates the majority of testing activities. We can 
conclude that the failures detected by MODEST are not trivial, since the set of volunteers did not detect all of them. Additionally, each injected fault generated a failure detected by at least one of the individual volunteers, showing that the injected faults were not specially contrived.

During our experiments we noticed that the number of generated test cases was comparable to the number of manually generated ones. We are planning to enlarge the size and the complexity of the systems in our experimental study, in order to evaluate MODEST scalability.

Currently, we are working in two different projects related to MODEST. We are developing a MODEST Mutation [17] Tool to help the evaluation of MODEST generated tests, and we are extending MODEST to deal with more sophisticated architectures.

\section{References}

1. NIST, Planning Report 02-3, The Economic Impacts of Inadequate Infrastructure for Software Testing, 2002, http://www.nist.gov/director/prog-ofc/report02-3.pdf, last access on November 2004.

2. Jacobson, I., Rumbaugh, J., and Booch, G., The Unified Software Development Process, Addison Wesley, 1999.

3. Rumbaugh, J., Jacobson, I., and Booch, G., The Unified Modeling Language Reference Manual, Addison Wesley, 1999.

4. Warmer, J., and Kleppe, A., The Object Constraint Language, Addison-Wesley, 2nd edition, 2003.

5. Maes, P., Concepts and Experiments in Computational Reflection. Proceedings of the Conference on Object-Oriented Programming Systems, Languages and Applications (OOPSLA'87), pages 147-155, Orlando, Florida, December 1987.

6. Kiczales, G., Lamping, J., Mendhekar, A., Maeda, C., Lopes, C., Loingtier, J., and Irwin, J., Aspect-Oriented Programming, In European Conference on ObjectOriented Programming (ECOOP'97), LNCS 1241, pages 220-242, Finland, June 1997.

7. OMG, UML Testing Profile, Object Management Group document, March 2003, available from http://www.omg.org, last access on November 2004.

8. OMG, XML Metadata Interchange (XMI). Object Management Group document, July 1998, available from http://www.omg.org/, last access on November 2004.

9. Basili, V., Selby, R., Hutchens, D., Experimentation in Software Engineering,IEEE Transactions on Software Engineering volume 12, number 7, July 1986.

10. Rational Software Corporation, Rational Rose User's Guide, available from http://www.rational.com/, last access on November 2004.

11. Abbott, Abbot Java GUI Test Framework, available from http://abbot.sourceforge.net/, last access on November 2004.

12. Offutt, J., and Abdurazik, A., Generating Tests from UML Specifications, Proceedings of the 2nd Unified Modeling Language Conference (UML'99), pages 416-429, Fort Collins, CO, USA, October 1999.

13. Briand, L., and Labiche, Y., A UML-Based Approach to System Testing, Proceedings of the 4th Unified Modeling Language Conference (UML'01) pages 194-208, Toronto, Canada, October 2001. 
14. Hartmann, A., and Nagin, K., The AGEDIS Tools for Model Based Testing, International Symposium on Software Testing and Analysis (ISSTA 2004) Boston, Massachusetts, USA, July 2004.

15. Hartmann, J., Imoberdorf, C, and Meisinger, M., UML-Based Integration Testing, Proceedings of the International Symposium on Software Testing and Analysis (ISSTA 2000), pages 60-70, Portland, Oregon, United States, August 2000.

16. Pickin, S., Jard, C., Le Traon, Y., Jéron, T., Jézéquel, J.-M., and Le Guennec, A., System Test Synthesis from UML Models of Distributed Software, Proceedings of 22nd IFIP WG 6.1 International Conference on Formal Techniques for Networked and Distributed Systems (FORTE 2002), pages 97-113, Houston, Texas, November 2002.

17. DeMillo, R., Lipton, R., and Sayward, F., Hints on Test Data Selection: Help for the Practicing Programmer, IEEE Computer, volume 11, pages 34-41, April 1978. 